Voix et Images

volxetimages

\title{
Des cailloux blancs pour les forêts obscures
}

\section{Anthony Purdy}

Volume 16, numéro 2 (47), hiver 1991

Jovette Marchessault

URI : https://id.erudit.org/iderudit/200899ar

DOI : https://doi.org/10.7202/200899ar

Aller au sommaire du numéro

Éditeur(s)

Université du Québec à Montréal

ISSN

0318-9201 (imprimé)

1705-933X (numérique)

Découvrir la revue

Citer cet article

Purdy, A. (1991). Des cailloux blancs pour les forêts obscures. Voix et Images, 16(2), 262-271. https://doi.org/10.7202/200899ar

Ce document est protégé par la loi sur le droit d'auteur. L'utilisation des services d'Érudit (y compris la reproduction) est assujettie à sa politique d'utilisation que vous pouvez consulter en ligne.

https://apropos.erudit.org/fr/usagers/politique-dutilisation/
Cet article est diffusé et préservé par Érudit.

Érudit est un consortium interuniversitaire sans but lucratif composé de l’Université de Montréal, l'Université Laval et l'Université du Québec à Montréal. Il a pour mission la promotion et la valorisation de la recherche. https://www.erudit.org/fr/ 


\title{
Des cailloux blancs pour les forêts obscures
}

\author{
par Anthony Purdy, Université de l'Alberta
}

Et, comme un oeil naissant couvert par ses paupières, Un pur esprit s'accroît sous l'écorce des pierres.

Nerval, *Vers dorés *

La Nature est un temple où de vivants piliers

Laissent parfois sortir de confuses paroles;

L'homme y passe à travers des forêts de symboles

Qui l'observent avec des regards familiers.

Baudelaire, *Correspondances *

Madame *** établit un piano dans les Alpes. La messe et les premières communions se célébrèrent aux cent mille autels de la cathédrale.

Rimbaud, *Après le déluge *

\section{Paratexte 1}

Dans le vieux cimetière d'Atlanta, on avait déjà enterré ses restes. Une petite pierre tombale en marbre gris. La terre fraîchement remuée. La réalité. Je me suis agenouillé.

Plus tard, quand jaai levé la tête, j’ai vu que quelqu'un me regardait au loin. C'était un ange de pierre, debout sur un socle en ciment. Debout dans la lumière brillante du milieu de cette matinée d'automne. Derrière lui, se dressait une rangée de magnolias où des oiseaux s'égosillaient. Tout était calme dans l'ombre bleutée de la nature. Partout, les feuilles d'automne

1 Tout en adoptant le terme de Gérard Genette, ma démarche dans cette première partie s'inspire davantage du genre d'analyse envisagé par Dominique Garand dans un article intitulé "La politique éditoriale comme contrat de lecture»: L'objet-livre n'est donc pas une simple médiation, un canal entre l'auteur et le lecteur, entre l'ecriture et la lecture, entre le discours et le marche, entre le texte et l'institution. Il est leur point de rencontre, leur carrefour agonique, le lieu, immédiatement offert au regard du lecteur, de leur rapport polémico-contractuel. Le 
tombaient lentement. Je regardais l'ange qui connaissait toutes ces tombes, tous ces morts. Pendañt des âges, il est demeuré ainsi, debout entre ciel et terre, pensai-je. Comme il m'attirait, m'aspirait vers lui. Peut-être que les anges de pierre attirent aussi bien les oiseaux que les esprits errants et désolés.

En m'approchant, je remarquai qu'une vigne' avait accroché ses vrilles sur le socle et sur les contours de l'ange. En grandissant, elles s'étaient resserrées sur lui dans une espèce d'étreinte végétale, comme si elles voulaient le retenir sur la terre. Pour la première fois de ma vie, je me tenais devant un ange. Dans le vieux cimetière d'Atlanta, cet ange de pierre tendait une main vers l'azur d'une manière délicate et vulnérable, pendant que l'autre main semblait plonger profondément dans la terre des morts. 2

Pour arriver à cette description de "l'Ange de la présence", il faut lire 150 pages du roman le plus récent de Jovette Marchessault, Des cailloux blancs pour les forêts obscures. Et pourtant, on a l'impression, en lisant ce passage, de reconnaître cet ange de pierre retenu sur la terre par ses chaînes vivantes, végétales. S'agirait-il d'un souvenir écran de quelque texte littéraire que l'on n'arrive pas à faire remonter jusqu'à la surface? D'une image entrevue quelque part, familière mais oubliée? Ce n'est qu'en refermant le livre que l'on finit par comprendre. Car là, sur la couverture (blanche), audessous du nom de l'auteure (en majuscules bleues) et du titre (en lettres noires) suivi de l'indication roman (également en noir), est la photo de l'Ange (voir page suivante). Au-dessous de la photo, ellemême en noir et blanc et encadrée de blanc, il n'y a que le logo (en gris) de la maison d'édition Leméac.

Une première lecture de la photo découvre un certain nombre d'oppositions binaires qui structurent nécessairement tout essai de déchiffrement. En premier lieu, les diverses oppositions du titre minéral/végétal, inanimé/animé, blanc/noir, clair/obscur - sont reprises de façon frappante sur le plan visuel, où l'on a l'impression d'assister à la métamorphose de la statue en être vivant (en vivant pilier, dirait le poète), tant l'étreinte végétale de la vigne semble insuffler la vie à la pierre inanimée. (Le motif des cailloux revient en noir et blanc à la page 44 du roman où la narratrice, Jeanne, raconte la

limiter au statut de médiateur laisserait supposer que le sens se produit ailleurs, que le livre est medium sans être message (dans E.D. Blodgett et A.G. Purdy, Préfaces et Maniféstes littéraires, Edmontón, Research Institute for Comparative Literature at the University of Alberta, 1990, sous presse.).

2 Jovette Marchessault, Des cailloux blancs pour les forêts obscures, Montréal, Leméac, 1987, p. 149-50. Toute' référence à cet ouvrage sera donnée entre parenthèses dans le texte de mon article. 


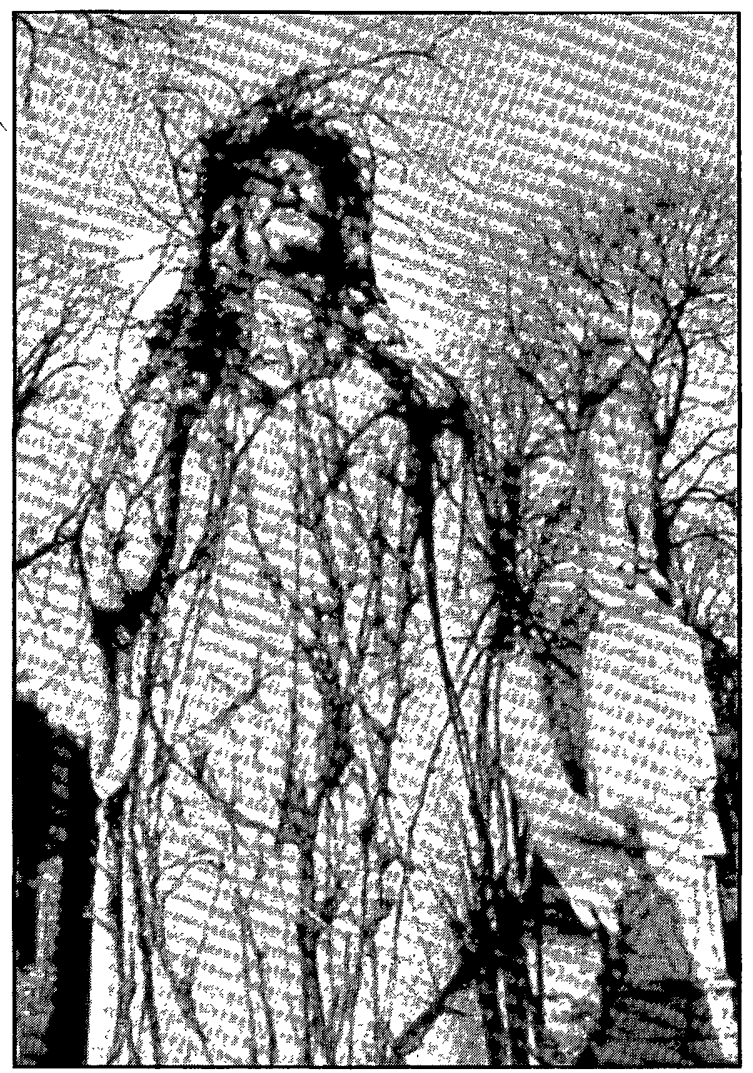

vision qui lui a été accordée du jugement dernier: Debout sur une poignée de terre rouge, je suis jugée sous les feux de la rampe par un ange solaire. Et c'est à travers mes larmes que je le regarde compter mes actes bénéfiques avec des cailloux blancs. Avec des cailloux noirs, mes actes nuisibles.) La présence contraignante de la vigne joue un rôle aussi dans l'opposition terre/ciel qui sera déterminante pour la thématisation du roman: contrariant le regard de l'ange qui est orienté vers le ciel, la vigne accentue le jeu des mains dont l'une est tendue vers l'azur d'une manière délicate et vulnérable, pendant que l'autre main [semble] plonger profondément dans la terre des morts (p. 150).

La photo, ainsi que la couverture dans son ensemble, se présente donc comme un lieu de confusion, d'ambiguïté et de conflit, impression qui est renforcée par l'incertitude en ce qui concerne le sexe de l'ange qui ne se laisse pas deviner à partir de la photo ${ }^{3}$. Mais si la

3 L'ambiguité du sexe se présente comme trait caractéristique traditionnel des anges dans un roman récent de Clotilde Martin, Gabriel penché: Les anges sont 
statue de la couverture est décidément androgyne, le texte du roman ne laisse flotter aucun doute quant au sexe de l'Ange de la présence, qui non seulement est lui-même masculin, mais aussi est explicitement assimilé au seul personnage masculin du roman, ce même "Lion de Bangor " qui se trouve agenouillé dans le cimetière d'Atlanta où sont enterrés les restes de sa femme: Il est semblable à toi et tu es semblable à Lui, lui souffle une voix qui ressemble à celle de Harriett, l'amante - morte depuis bien des années - de son épouse défunte (p. 150). Une telle assimilation de l'androgyne au masculin me semble d'autant plus curieuse et ambiguë qu'il s'agit d'un texte qui vient apparemment s'insérer dans une lignée romanesque que l'on a l'habitude de qualifier de féministe et lesbienne.

Une deuxième photo, d'Algis Kemezys comme celle de la statue, se trouve sur la quatrième de couverture. Placée à gauche et en bas, et plus petite que la photo de l'ange, elle montre la tête et les épaules de l'auteure, qui est peut-être assise sur ses talons, devant l'une de ses sculptures amérindiennes. (La même photo, mais plus petite encore et sans sculpture identifiable parce que tout a été coupé au profit du visage, figure sur la couverture de la pièce de théâtre de Jovette Marchessault, Demande de travail sur les nébuleuses, Leméac, 1988. Chose curieuse : ce sont les romans qui, en tant qu'objets visuels, exploitent le rapport entre écrivaine et sculpteure, rapport que les pièces de thêatre ont tendance à taire. Comment expliquer cette suppression d'une partie importante de la signature professionnelle et artistique de Jovette Marchessault que représente la réduction de la photo?) La sculpture ressemble par son style à celle — * La Mère des herbes - Femme tellurique " - qui figurait sur la couverture du roman la Mère des herbes ${ }^{4}$. Par là, la photo annonce la filiation du roman de 1987 avec les précédents, car il s'agit bien du dernier volet de la trilogie Comme une enfant de la

par essence incorporels. Visibles, ce sont des êtres photographiques, des instantanés: des yeux sans regard, des lères sans paroles, des corps sans mouvement. Représentation artistique imparfaite, ils se glissent dans nos tableaux, dans nos songes, a la porte des églises, toujours muets, aveugles, immobiles. Une ou plusieurs paires d'ailes, l'ambigulte du sexe les caracterisent. Invisibles, ils nous laissent cette pudeur qui ne trompe pas, cette rougeur tranquille, la fadeur de l'air, comme un bruissement, une chute de petales: on dit qu'un ange passe. (Clotilde Martin, Gabriel penché, Paris, Editions du Seuil, 1988, p. 7.) Cf. Des cailloux blancs pour les forêts obscures, p. 19.

4 Jovette Marchessault, la Mère des herbes, Montréal, Quinze, 1980 (Réelles). Comment expliquer ce déplacement, du deuxième roman au troisième, de la sculpture amérindienne - et de la nouvelle mythologie du Grand Esprit mère qu'elle represente - de la couverture avant à la couverture arrière? Et le fait que "La mère des herbes " ait été remplacée par "l'Ange de la présence ", statue des plus classiques, d'inspiration nettement européenne, peut-être du XIX siècle, et qui represente, parait-il, un personnage du sexe masculin? Autant d'enigmes qu'il s'agit d'explorer. 
terre $^{5}$. Cependant, cette filiation est occultée et par la liste des ouvrages de la même auteure - où figure la référence doublement erronée à Comme une enfant de la terre, roman, Montréal, Leméac, 1974 (Prix France-Québec) - et par le petit texte qui accompagne la photo sur la quatrième de couverture: Peintre, sculpteure, romancière et dramaturge, Jovette MARCHESSAULT s'est notamment illustrée par la célébration des grandes figures littéraires $d u X X^{e}$ siècle, dont les biographies et les œuvres lui ont inspiré des textes majeurs de notre littérature dramatique. Cette promotion du théâtre de l'auteure aux dépens de sa production romanesque est sans doute attribuable au fait que la distance temporelle entre les trois volets de la trilogie (1975-1980-1987) est trop grande pour que celle-ci soit un facteur exploitable dans la commercialisation du dernier roman, tandis que les pièces de théâtre de Marchessault auraient créé un public - et donc un marché - au cours des années 1980. En effet, la seule mention de la trilogie romanesque se trouve dans le texte de Gloria F. Orenstein qui figure également sur la quatrième de couverture dont il occupe plus que la moitié.

Le rôle joué par Gloria Orenstein dans la production du discours paratextuel de la trilogie romanesque - et, partant, dans la réception et dans l'institutionnalisation de celle-ci 6 - est si important qu'il vaut peut-être la peine de citer le texte entier:

Dans ce roman du troisième oil, qui inaugure la littérature visionnaire de l'ère du Verseau, les forces de la lumière et de l'amour triomphent des forces de l'obscurité et du mal; on y trouve une véritable et tendre réconciliation entre femmes et hommes, enfants et parents, animaux et humains, passé et avenir, portée par une vision chamaniste de l'interconnexion du visible et de l'invisible.

Ce dernier volet de la trilogie Comme une enfant de la terre met en scène Noria, une aviatrice qui, telle une chamane, vole à travers le ciel nocturne de l'Amérique du Nord pour livrer au monde un message de salut et un espoir de guérison, et Jeanne, l'écrivaine qui pratique son propre art magique de la guérison: l'art de la littérature.

Le roman de Jovette Marchessault déplace une masse critique de lumière et d'élan vital suffisante pour "attirer" l'âme,

5 Le premier roman de la trilogie, le Crachat solaire, parut en 1975, chez Leméac.

6 Limportance capitale du discours paratextuel d'Orenstein dans la légitimation de l'cuvre romanesque de Marchessault ainsi que dans le mode d'insertion de celleci dans un habitus particulier, vient étayer l'hypothèse de Dominique Garand, selon laquelle ['Jinstitution est sécrétée dans le type de contrat qui s'établit entre le texte et l'appareil éditorial qui le prolonge, d'autant plus que ce contrat est le plus souvent perturbe (parce qu'issu) d'eléments conflictuels: il faut orienter le texte de façon à le rendre institutionnellement recevable (loc. cit.). 
captiver l'esprit et donner accès aux sources anciennes du savoir sacré. L'énergie de son écriture active de nouvelles vibrations, charge les résonances cosmiques de fréquences nouvelles et incite à une révision du mythe de la création, le récit sublime de nos origines personnelles et karmiques.

La voix de cette auteure est celle de la renaissance cosmique... Une voix dont la longueur d'onde est un rayon d'Amour.

Or comment recevoir ce texte court qui, par ce qu'il dit, est largement redondant, par ce qu'il tait, tout simplement étonnant? Redondant parce qu'il s'agit d'une rhétorique que les lectrices et les lecteurs de Jovette Marchessault connaissent déjà par les autres écrits d'Orenstein, y compris la préface à la Mère des herbes ${ }^{7}$ : rhétorique du nouvel âge, d'un féminisme essentialiste et mystique qui réduit le texte littéraire à ses dimensions mythologique et religieuse. Étonnant parce qu'il laisse entièrement de côté la moitié au moins du roman, car, comme l'a signalé Louise Milot dans un compte rendu ${ }^{8}$, Des cailloux blancs pour les forêts obscures n'est pas un texte homogène mais, au contraire, comprend deux romans bien distincts: d'une part, le roman de Jeanne et de Noria - de l'écrivaine et de l'aviatrice - et de la communauté lesbienne des Appalaches où se déroule leur histoire; d'autre part, le roman familial de Noria, l'histoire de sa mère et, surtout, de son père, ce fameux Lion de Bangor qui raconte à Jeanne une bonne partie de cette histoire.

Avec, sans doute, les meilleures intentions du monde, le discours d'Orenstein récupère le roman de Marchessault en l'aplatissant et en l'homogénéisant, en supprimant les conflits et les ambiguïtés qui existent dans le texte du roman et qui sont affichés par certains des éléments paratextuels que je viens de signaler ${ }^{9}$. À cet égard, il me semble que la critique américaine, cofondatrice du "Women's Salon * de New York, a joué le rôle d'interprêtresse (pour féminiser une expression de Jean-Marc Lemelin) dans la réception de la trilogie ainsi que dans la production du sens de ces romans: c'est-à-dire qu'elle a transformé des textes littéraires en objets de culte tout en prétendant en livrer la vérité - cohérente, unifiée, sans faille - à un public qu'elle voudrait ainsi métamorphoser en congrégation. Le

7 Outre la preface à la Mère des herbes dont un extrait figure également sur la quatrième de couverture du même roman, voir surtout Gloria Orenstein, "Jovette Marchessault ou la quête extatique de la nouvelle chamane féministe w, Bulletin de la Société des profeseeurs français en Amérique, I, automne 1979, p. 37-57.

8 Louise Milot, “Un homme parmi les femmes », Lettres québécoises, ${ }^{\circ} 48$, hiver 1987-88, p. 18-20.

9 Cf. Garand, loc. cit. : la démarche contractuelle soutenue par la politique éditoriale n'a de pertinence que parce que sont mises en contact, au sein de l'objet-livre, deux instances conflictuelles: le texte comme système d'ecriture et l'archi-texte comme procès de lecture. 
texte, tout comme le paratexte, du troisième roman de Marchessault est un lieu de conflits et de contradictions, ce que le discours institutionnel - essentialiste, totalisant, monologique - de Gloria Orenstein s'efforce de masquer. Essayons donc d'ouvrir ce texte clos pour en faire ressortir quelques-uns de ces éléments conflictuels. Je m'attacherai en premier lieu à la figure de l'écrivaine dont le statut dans le roman est explicitement problématique.

\section{Texte}

Jeanne, lesbienne du vingtième siècle (p. 13) et narratrice du roman, est tout à fait consciente des ambiguités de sa pratique d'écrivaine, qui participe à la fois de la douce extase du projet mystique et de l'exercice du diable, de la prostitution littéraire de l'expérience intime, de la lutte quotidienne avec la langue dans toute sa matérialité:

Qu'est-ce qu'une écrivaine? Quelqu'une qui se joue constamment des scènes et qui prend le premier café du matin avec des bribes de phrases effilochées dans sa tête. Quelqu'une qui se promène dans l'énergie du monde avec laquelle elle converse, s'exaspère et rompt avec fracas. (p. 36)

Elle se trouve constamment aux prises avec les limites de la littérature et de sa propre écriture - Ecrire. Donner un coup d'épée dans l'air. Faire une crise de nerfs dans le ventre d'une carpe! (p. 14) mais reste quand même convaincue des possibilités utopiques et révolutionnaires de l'entreprise: Si, pour une fois, elle se permettait de penser au-delà de ce qu'on lui permet de penser, la littérature (p. 14). En effet, malgré ses doutes et ses incertitudes, le rôle qu'elle se donne en tant qu'artiste est un rôle exalté qui la rattache à toute une lignée d'artistes visionnaires:

Moi, infime créature qui a peur de tout, je viens de me rappeler que mon rôle, un rôle que je connais par cœur mais que j'avais oublié dans le fond de mon enfance, est de sauver le monde. Ce rôle, j'en suis persuadée, nous le savons tous et toutes. Même si nous essayons de nous en distraire et de nous en défaire à jamais par des performances prodigieuses, ou par des rêves de vengeance, de bonheur et de gloire. Notre rôle est de sauver ce vieux monde si beau, si cruel et si tendre, que nous aimons à travers nos générations sans nombre, nos artistes et nos visionnaires. (p. 12)

Mais, pour assumer pleinement ce rôle de voyante, elle a dû rompre en quelque sorte avec l'institution en quittant Montréal et en s'établissant dans les Appalaches: Montréal... J'y ai vécu enfoncée à miflanc dans la peur. Pour conserver ma petite notoriété, j’ai fait les gestes qu'il fallait faire. Ce qui est fascinant, dans les milieux de la culture, c'est que tout le monde traite tout le monde sur un pied 
d'égalité: c'est-à-dire comme un décor, le décor de son propre ego (p. 30). En quittant la ville, elle a l'impression de renaître à la vie et de déjouer ainsi le triste sort de l'écrivain:

- À Montréal, j’habitais surtout une petite notoriété littéraire, n'intéressant que les intéressés, dis-je en riant. Et chaque notoriété, grande ou petite, est à son tour logée sur un continent littéraire. Ce continent littéraire fait irrésistiblement penser à une cité minière. Chacun, chacune y travaille, creusant son trou, hurlant le nom de son filon et ses droits de propriétaire. Avec des coups de pioches, de pelles, de dents, les taupes de fer s'enfoncent dans la mine.

Un jour je creusais férocement mon trou, parmi les autres, ma pioche a fait éclater - je l'avais frappé en pleine poitrine - le corps momifié d'un écrivain oublié depuis longtemps. Il avait été porté disparu! Même si le temps et l'oubli l'avaient réduit à la fine proportion de la peau sur les os, j'ai reconnu l'odeur de pourriture. J'ai identifié les atomes grandissants et bientôt permanents de mon futur. Une véritable force d'explosion m'a balayée hors de la ville minière. (p. 15)

Cependant, en échangeant cette existence industrielle souterraine contre l'indépendance et la liberté de la vie dans les montagnes, Jeanne retrouve sa chère vieille garde lesbienne montréalaise qui avait autrefois fondé des revues, des maisons d'édition, des archives lesbiennes (p. 54) avant de se réfugier dans la petite communauté des Appalaches. Ces femmes, les cigales rouges selon le nom que Jeanne leur donne dans son cour. ${ }^{10}$, constituent, comme l'a suggéré Louise Milot, une sorte de chœur antique dont le rôle nous est depuis longtemps familier:

Y a-t-il une écrivaine du Québec, depuis les quinze dernières années, qui n'ait pas eu recours, de quelque façon et à un moment ou l'autre, à la collectivité des femmes anonymes - ses mères, ses sœurs, ses filles - ou des femmes célèbres - du passé ou du présent - pour se protéger, se défendre, s'affirmer, pour tout simplement se dire? ${ }^{11}$

Mais ce qu'il y a d'intéressant dans cette convocation d'un discours collectif et "maternant " de femmes - et dont la critique assez dure

10 Le nom de «cigales rouges * renvoie au même cycle d'ensevelissement et de renaissance qu'a connu Jeanne et que celle-ci décrit en termes typiquement telluriques: Ce n'est qu'après avoir séjourné, comme le font toutes les cigales, dixsept années dans le sol, parmi les minéraux et les racines, qu'elles surgissent enfin de leur écrin de terre pour craqueter dans l'air ce chant torride qu'elles ont si longtemps couvé dans l'humus et l'obscurité. Est-ce cette longue détention dans la terre qui donne une telle fougue à leur chant? (p. 47).

11 Louise Milot, loc. cit., p. 18. 
de Milot ne semble pas tenir compte - c'est le rapport fort équivoque qui s'établit dans le texte entre cette voix communautaire et celle de la narratrice, entre Jeanne et ses lointaines voisines (p. 47).

En effet, tout au long du roman, pour évoquer la communauté lesbienne, la narratrice adopte un ton de tendresse mêlée d'ironie ${ }^{12}$ :

Ce qui étonne chez ces athées et ces séparatistes, c'est qu'elles semblent avoir importé de "chez les hommes» des pratiques religieuses réglées par un rite: celui de porter avec ostentation la veste de cuir, de daim ou de tweed. La cravate ou le nceud papillon, les bottines militaires, le cheveu court et la lotion après rasage. Ce sont nos chevalières, les grandes "dykes" de l'Amérique du Nord. Nos chevalières aiment voyager à travers le continent en quête d'aventures féminines. Chevauchant les juments dociles de leur cheptel vif, elles vont, brandissant l'épée flamboyante de l'autonomie. Le regard et le muscle durs, l'attitude un peu faunesque, cherchant à sillustrer par un lignage ancien, elles prennent plaisir à faire crisser dans l'air le labyris, cette hache à double tranchant de la légende amazonienne, en chantant d'une voix grave la bonne parole de la révolte sorcière, ainsi qu'une musique portée par les vents. (p. 47-48)

L'attitude de méfiance critique de la part de Jeanne à l'égard des cigales rouges remonte peut-être à un incident particulier qui a eu lieu une nuit qu'elle s'est réveillée d'un mauvais rêve pour aller chez elles chercher du secours:

J'allais frapper à la première porte pour demander un peu de réconfort, quand j'ai entendu des bruits alarmants qui jaillissaient des murs de vos maisons. Quelqu'un déplaçait des meubles... Quelqu'un donnait des coups suivis de cris de douleur étouffés... Une femme battait une autre femme, lui obstruant la bouche pour l'empêcher de souffrir à voix haute... Je ne savais pas quoi faire de cette souffrance et je me sentais si près de cette scène, de l'autre côté dé la porte. (p. 109)

Sa désillusion était telle que, incapable de supporter la complication d'une communauté lesbienne utopique souillée de brutalité et de cruauté, elle est restée par la suite quelque peu à l'écart du collectif, ce que la vieille garde s'expliquait en termes de sa condition quasi monastique d'écrivaine.

En évoquant rapidement les rapports complexes qui existent entre ces deux lieux conflictuels - l'institution littéraire et la

12 L'ironie s'étend aussi à tel aspect du discours du nouvel áge. Par exemple, quand on demande à Jeanne quel est son signe zodiacal, elle répond qu'elle est Verseau, ascendant Lionne et soumise d̀ des déséquilibres périodiques. - Comme la Russie, ironise une voix (p. 62). 
communauté lesbienne - je suis loin d'avoir épuisé les ambiguités et les contradictions du roman de Marchessault. Il faudrait, par exemple, se pencher sur ces touchantes histoires d'amour entre Jeanne et Noria, d'une part, et la mère de celle-ci et Harriet d'autre part. Il faudrait examiner le comportement fort équivoque de cette dernière, ainsi que le rôle inquiétant joué par le spectre de Mary Lane et les femmes du Ku Klux Klan, les Filles Unies de la Confédération. Il faudrait s'attacher au caractère du Lion de Bangor et à son mariage avec la mère de Noria. Et surtout, il faudrait s'interroger sur le portrait de ce * Père des pères * et sur le rapport père-fille qui est peut-être en fin de compte le plus émouvant de tous dans ce texte. Mais tout cela exige que l'on abandonne une fois pour toutes la perspective réductrice, unifiée, faussement cohérente, dans laquelle Jovette Marchessault romancière a été enfermée jusqu'ici. Un texte littéraire n'est jamais le produit d'un seul code; il est temps que les romans de Marchessault entrent dans la littérature et que l'on propose des codes de lecture capables de rendre compte de leur complexité, de leur hétérogénéité. Le féminisme de l'auteure ainsi que la compassion qui le nourrit, ne souffriront pas de la mise en évidence de la polyphonie du véhicule ${ }^{13}$.

13 Une lecture attentive à la polyphonie du texte est exigée par le roman lui-même, comme l'atteste la leçon du huart dont le répertoire de cris variés échappe encore a la science des ornithologues (p. 55-56). 